Radial and Nonradial Pulsations as Probes of Stellar Physics

ASP Conference Series, Vol. 259, 2002

C. Aerts, T.R. Bedding, \& J. Christensen-Dalsgaard, eds.

\title{
Search for a Magnetic Field in the B2 IV Star V 2052 Oph
}

\author{
C. Neiner ${ }^{1}$ \\ Observatoire de Paris-Meudon, UMR 8633 CNRS, France 8 \\ Sterrenkundig Instituut Anton Pannekoek, University of Amsterdam \\ H.F. Henrichs, A. Tijani, V. Geers \\ Sterrenkundig Instituut Anton Pannekoek, University of Amsterdam
}

\section{J.S. Nichols}

Harvard/Smithsonian Center for Astrophysics, Cambridge MA, USA

\begin{abstract}
We have searched for magnetic signatures in the B2 IV star V 2052 Oph, a $\beta$ Cep star with stellar wind behavior typical for an oblique magnetic rotator, as $\beta$ Cep itself. From UV spectroscopy we determined an accurate rotational period and $v \sin i$, and present the magnetic measurements as a function of rotational phase. The (small) class of magnetic pulsating early B stars are of great asteroseismological interest.
\end{abstract}

\section{Introduction}

The B2 IV star V 2052 Oph is a $\beta$ Cep variable with a $3^{\mathrm{h}} 21^{\mathrm{m}}$ pulsation period. The variability of its UV wind lines is typical for oblique magnetic rotators (Henrichs et al., 1998; Henrichs, 2001), as was convincingly demonstrated for $\beta$ Cep itself, which shows similar stellar wind variability and hosts a dipole field of about 300 G (Henrichs et al., 2001; Donati et al., 2001). So far, only three early B stars are known with this type of signatures. Analysis of the pulsation properties of a rotating magnetic star gives very strong constraints on its parameters and its evolutionary stage, which is of great asteroseismological interest.

\section{UV and spectropolarimetric observations}

UV Spectroscopy: We obtained 41 IUE spectra (1150-2000 $\AA)$ during 1981-82 and 1994-95. The CIV, SiIV and NV wind lines are strongly modulated at $3.63890 \mathrm{~d}$, which we identify as the rotational period. From the photospheric UV lines we determined $v \sin i=85 \mathrm{~km} / \mathrm{s}$, implying $R_{*}=6.1 R_{\odot}$ and $i=80^{\circ}$, using a distance of $254 \mathrm{pc}$ and a luminosity of $L=3.74 L_{\odot}$. The C IV equivalent width as a function of rotational phase is plotted in Fig. 1b. As in $\beta$ Cep (Fig. 1a), there is one deep minimum (i.e., maximum emission) and two unequal maxima.

${ }^{1}$ e-mail: Coralie.Neiner@obspm.fr 

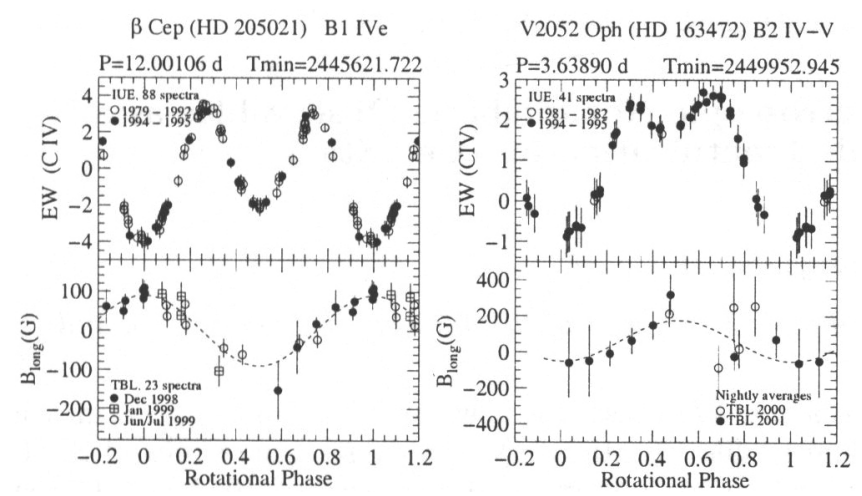

Figure 1. The magnetic star $\beta$ Cep and the candidate V $2052 \mathrm{Oph}$.

Spectropolarimetry: Observations were obtained with the MUSICOS echelle spectropolarimeter $(R=35000,4500-6600 \AA)$ at the $2 \mathrm{~m}$ Telescope Bernard Lyot at Pic du Midi, France in 2000-01. A least-squares deconvolution (Donati et al., 1997) allows the detection of a stellar magnetic field (longitudinal component only) through the Zeeman signatures generated in the Stokes $V$ line profiles. Due to the relative faintness of the star, the individual measurements have large error bars. In Fig. $1 \mathrm{~b}$ we present the nightly averages of 1-5 magnetic measurements.

\section{Results and conclusions}

Although no strong signatures appear in the individual Stokes $V$ profiles, the varying nightly averages point towards the presence of a magnetic field. A bestfit sine wave gives an amplitude of $B_{l}=114 \pm 48 \mathrm{G}$, with an average value of $B_{0}=$ $63 \pm 30 \mathrm{G}$ and reduced $\chi^{2}=1$. This is consistent with a rotationally modulated magnetic dipole field, but measurements with a better $\mathrm{S} / \mathrm{N}$ are needed. Note, however, the striking resemblance in phase correlation with $\beta$ Cep. From the phase difference between the UV equivalent-width maxima we derive $65^{\circ}<i<$ $82^{\circ}$, and $45^{\circ}<\beta<17^{\circ}$ for the angle of the magnetic axis.

New generation spectropolarimeters like Espadons are needed to confirm the detection of a field in V $2052 \mathrm{Oph}$. Such magnetic pulsating stars are apparently rather rare, but will provide the most massive examples of stars useful for asteroseismological (and hence evolutionary) tests.

\section{References}

Donati, J.-F., Semel, M., Carter, B., et al. 1997, MNRAS 291, 658

Donati, J.-F., Wade, G.A., Babel, J., et al. 2001, MNRAS, 326, 1265

Henrichs, H.F., de Jong, J.A., Kaper, L. et al. 1998, ESA-SP 413, 157

Henrichs, H.F. 2001, in Proc. ESO Workshop Santiago, ed. G. Matthys, in press Henrichs, H.F, de Jong, J.A., Donati, J.-F., et al. 2001, A\&A, submitted 